\title{
MATHEMATICAL PEARLS
}

The purpose of this department is to publish very short papers of an unusually elegant and polished character, for which there is normally no other outlet.

\section{FREE PRODUCTS WITH AMALGAMATION AND 3-MANIFOLDS}

D. B. A. EPSTEIN

Throughout this paper $M$ will be a compact 3-manifold, possibly with boundary.

If $M$ is a closed 3-manifold and $\pi_{2}(M)=0$, then $\pi_{1}(M)$ is not a nontrivial free product. This widely known result can be deduced from the theory of ends $[1$, p. $100 ; 2$, Satz VI]. A generalisation of the result is proved here, without using ends.

Lemma. If $\pi_{1}(M)$ is infinite and $\pi_{2}(M)=0$, then $M$ is aspherical.

This can be seen by using the Hurewicz isomorphism theorem in the universal cover of $M$.

THEOREM. Let $\pi_{1}(M)=A *_{C} B(C \neq A, B)$, i.e. a nontrivial free product with amalgamation. Let $\pi_{2}(M)=0$. Then, for any simple coefficient group $\Lambda$,

$$
H_{3}(A ; \Lambda)=H_{3}(B ; \Lambda)=H_{3}(C ; \Lambda)=0
$$

and $H_{2}(C ; \Lambda)$ contains a subgroup isomorphic to $H_{3}(M ; \Lambda)$.

Since $\pi_{1}(M)$ is infinite, we know from the lemma that $M$ is aspherical. Therefore, in the following diagram of covering spaces, all spaces are aspherical.

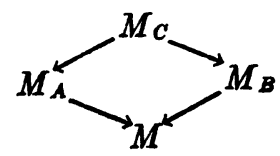

where $\pi_{1}\left(M_{G}\right) \approx G, G=A, B$ or $C$. The groups $A, B$ and $C$ each have infinite index in $A *_{C} B$. Therefore $M_{A}, M_{B}$ and $M_{C}$ are infinite sheeted coverings of $M$. So $H_{3}(G ; \Lambda) \approx H_{3}\left(M_{G} ; \Lambda\right)=0$ where $G=A, B$ or $C$.

We construct the mapping cylinders of $M_{A} \leftarrow M_{C}$ and $M_{C} \rightarrow M_{B}$ and identify the two subspaces $M_{C}$. Call the resulting space $M^{\prime} . M^{\prime}$ is aspherical, as can be seen using the Hurewicz isomorphism theorem

Received by the editors February 16, 1961. 
and the Mayer-Vietoris sequence in the universal cover of $M^{\prime}$. Also $\pi_{1}\left(M^{\prime}\right) \approx A *_{C} B$ by van Kampen's theorem. Therefore $M^{\prime}$ is homotopy equivalent to $M$. From the Mayer-Vietoris sequence for $M^{\prime}$, $M_{A}, M_{B}$ and $M_{C}$, we have the exact sequence

$$
0 \rightarrow H_{3}\left(M^{\prime} ; \Lambda\right) \rightarrow H_{2}\left(M_{C} ; \Lambda\right) .
$$

The theorem follows.

Corollary. If $M$ is closed and $C=1$, then $\pi_{2}(M) \neq 0$.

For $H_{2}\left(C ; Z_{2}\right)=0$ and $H_{3}\left(M ; Z_{2}\right)=Z_{2}$.

\section{REFERENCES}

1. H. Hopf, Enden offener Räume und unendliche diskontinuierliche Gruppen, Comment. Math. Helv. vol. 16 (1943) pp. 81-100.

2. E. Specker, Die erste Cohomologiegruppe von Überlagerungen und HomotopieEigenschaften dreidimensionaler Mannigfaltigkeiten, Comment. Math. Helv. vol. 23 (1949) pp. 303-333.

Princeton University 